\title{
Acute hemorrhagic edema of infancy - is it really a mild, benign disease?
}

\author{
Rasa Medovic ${ }^{1,2}$, Marija Medovic ${ }^{3,4}{ }^{4}$, Zoran Igrutinovic ${ }^{1,2 \oplus}$, Aleksandar Vlahovic ${ }^{5,66}$, \\ Gordana Kostic ${ }^{1,2 \oplus}$, Gordana Ristic ${ }^{3,4}$, Biljana Vuletic ${ }^{1,2 \oplus}$, Slavica Markovic ${ }^{1,2}$ \\ ${ }^{1}$ Pediatric Clinic and ${ }^{4}$ Center for Dermatovenerology, Clinical Centre Kragujevac, Serbia; Departments of ${ }^{2}$ Pediatrics and \\ ${ }^{3}$ Dermatovenerology, University of Kragujevac, Faculty of Medical Science, Serbia; ${ }^{5}$ Department of Plastic and Reconstructive Surgery \\ and Burns, Institute for Health Protection of Mother and Child of Serbia, Belgrade, Serbia; ${ }^{6}$ Department of Surgery, University of \\ Belgrade Faculty of Medicine, Serbia.
}

\begin{abstract}
Background. Acute hemorrhagic edema of infancy (AHEI) is a rare vasculitis, which presents dramatically as palpable purpuric skin lesions on the limb, face and auricles, with swelling of these parts and low-grade fever, in children up to 2 years of age. To date, approximately 400 cases have been described in the literature. The etiology remains mostly unknown. With or without treatment, AHEI goes to spontaneous recovery within 1-3 weeks, usually without any complications. To our knowledge, compartment syndrome as complication of AHEI has only been reported in one case. We present an unusual case of AHEI with serious complications due to compartment syndrome of the right-hand fingers.

Case. A 16-month-old male child presented with fever and sudden appearance and rapid spread of palpable, painless, non-itching ecchymotic hematomas on the thigh, cheeks, earlobes, forearms, dorsum of hands and feet, with mild edema of these regions. Complete systemic examination and all vital parameters were normal for age. There was no history of bleeding disorders in the family. Except low hemoglobin on complete blood count and increased D-dimer values, all other laboratory investigations were in the normal range.

Changes on the right forearm and hand expanded on almost the entire dorsal side and all surfaces of the fingers, with pronounced swelling and formation of bullous lesions, which were spreading and cracking. Skin biopsy confirmed nonspecific small-vessel vasculitis. That required the use of Methylprednisolone, low-molecularweight heparin, antibiotics and debridement of necrotic eschar, with necrectomy of the affected fingers.
\end{abstract}

Conclusions. Early recognition of AHEI is important to avoid unnecessary investigation and therapy. On the other hand, our reported case warns that unexpected complications may occur.

Key words: acute hemorrhagic edema, infancy, compartment syndrome.

Acute hemorrhagic edema of infancy (AHEI) is a rare form of hypersensitivity reaction which occurs in children up to 2 years of age. ${ }^{1,2}$ It presents as sudden non-itching, painless localized palpable purpuric targetoid skin lesions of the extremities, face and auricles, followed by limb and facial swelling and lowgrade fever. Children are usually minimally

$凶$ Zoran Igrutinovic

igzor@medf.kg.ac.rs

Received 26th December 2019, revised 12th February 2020, 27th May 2020, accepted 30th May 2020. affected. ${ }^{3}$ AHEI is vasculitis of small dermal vessels, characterized by inflammation with fibrinoid necrosis and extravasation of red blood cells. ${ }^{2,4}$ The etiology is mostly unknown. ${ }^{4-6}$ In the majority of patients, laboratory analyses are within normal values. ${ }^{4,7}$ Treatment with antihistamines and/or corticosteroids have been reported, but is still controversial. . $^{3,7-9}$ According to most authors, this is unnecessary due to its spontaneous recovery within 1-3 weeks, usually without any complications. ${ }^{4,7,8,10}$ Less than $10 \%$ of patients have extra cutaneous manifestations. ${ }^{7,10-15}$ 
Our case of AHEI had serious complications due to compartment syndrome of the righthand and fingers which formed necrotic eschar on affected parts of the right forearm. To the best of our knowledge, so far, only one case of AHEI and compartment syndrome has been described. ${ }^{16}$ Our patient required application of the low-molecular-weight heparin and debridement of necrotic parts. Unfortunately, necrotomy of the affected fingers had to be performed.

\section{Case Report}

A 16-month-old male child was admitted to the Pediatric Clinic on the second day of his complaints, with a history of fever $\left(38.2^{\circ} \mathrm{C}\right)$ and sudden appearance of ecchymotic purpura on both sides of the thighs and tiny petechial changes on the earlobes. Described lesions

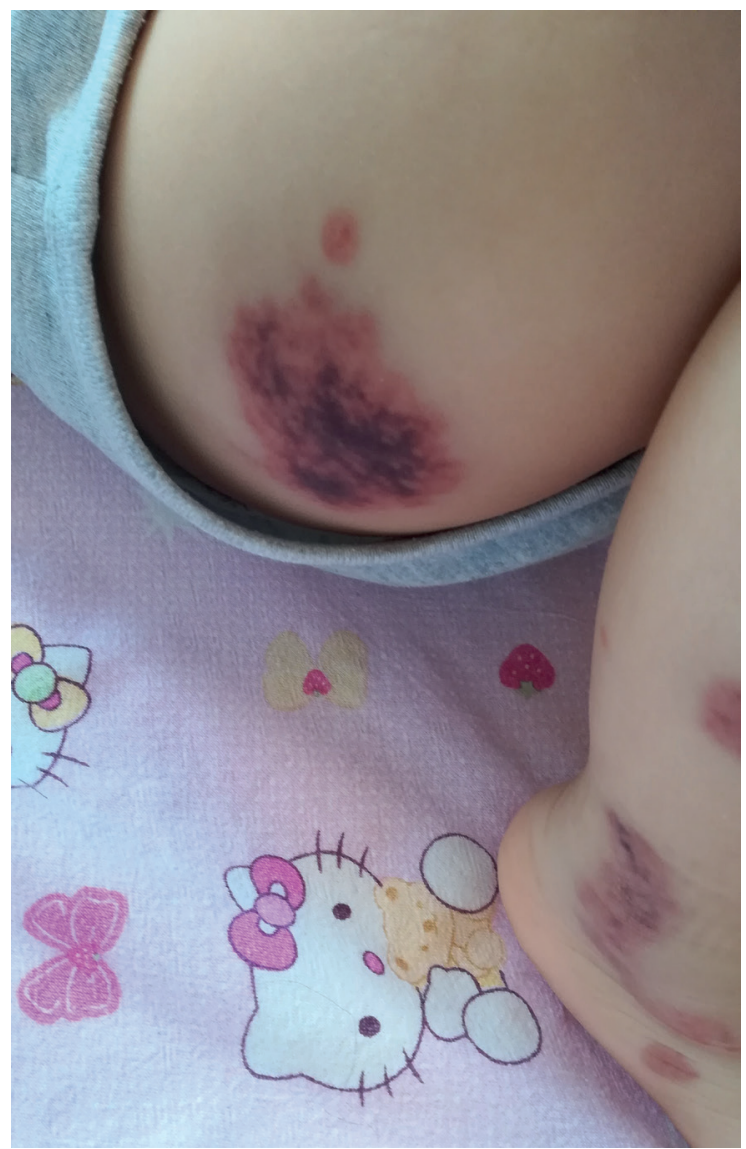

spread rapidly, with the appearance of new similar changes on the cheeks, lower legs and forearms, as well as on the dorsum of the hands and feet, with mild edema of these regions and all fingers. Some changes appeared as hematomas, with dimension between 0.5 and $10 \mathrm{~cm}$ in diameter, and individual changes as palpable, painless, non-itching, clearly limited lesions, of similar dimensions, with necrotic parts in the center and purple periphery (Fig. 1 and 2). Swelling of the left earlobe was also noticed, as well as tiny petechial changes on buccal mucosa, which were not extensive and did not disturb feeding. In the primary care, the patient was treated only with Paracetamol.

On admission, except for the described symptoms, complete systemic examination was normal and all vital parameters were stable. Otherwise, the child was delivered from regular full term pregnancy, with

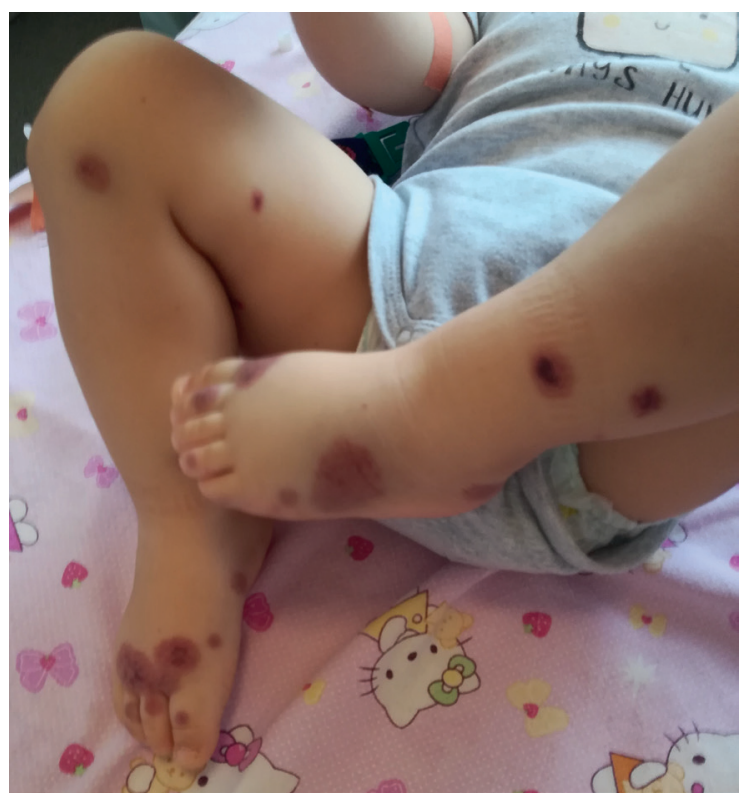

Fig. 1 and 2. Hematomas and ecchymotic purpuric skin lesions on different parts of the body. 
normal psychomotor development, and was age appropriately vaccinated (the last vaccine against Measles, Mumps, and Rubella was three months prior). He was circumcised two months before admission. He had also suffered from viral gingivostomatitis 20 days before the onset of the current disease. Buccal mucosa was treated with symptomatic local therapy only and fully recovered within 10 days. For the past four months, anemia had been treated with iron supplementation. There was no history of bleeding disorders in the family.

Initial laboratory investigations showed anemia on complete blood count (CBC) (hemoglobin $88 \mathrm{~g} / \mathrm{L}$, ferritin $6 \mathrm{~g} / \mathrm{L}$, serum iron $2.0 \mu \mathrm{mol} / \mathrm{L}$ ), with normal number of erythrocytes, platelets and leucocytes. The patient had normal blood coagulation screening (activated partial thromboplastin time (aPTT), prothrombin time (PT) and fibrinogen), markers of inflammation (C-reactive protein and procalcitonin), erythrocyte sedimentation rate, liver and kidney function tests, electrolytes, serum proteins and albumins, values of immunoglobulins and complements and urine analysis. The only parameter that was beyond the reference values was D-dimer $(7.21 \mathrm{ng} / \mathrm{ml})$ (Table I). Also, urine and blood cultures, virology analyses (Epstein Barr, Cytomegalovirus, Herpes viruses, Adenovirus, Parvo B19, Hepatitis B and C, HIV) and Immunoglobin $\mathrm{M}$ (IgM) on Mycoplasma pneumonia were all negative. Tests for congenital (protein $\mathrm{C}$ and $\mathrm{S}$, antithrombin III (AT III), activated protein $\mathrm{C}$ resistance $\mathrm{V}$ (APCR-V) assay, homocysteine - Table I) and acquired thrombophilia (lupus anticoagulant, anticardiolipin and beta-2-glycoprotein I antibodies), hormones and antibodies of the thyroid gland, antinuclear antibodies, antistreptolysin $\mathrm{O}$, anti-myeloperoxidase, anti- proteinase 3 antibodies and rheumatoid factor were within the normal range. Ultrasound examinations of the abdomen excluded visceral involvement.

After rapid assessment and exclusion of other clinical conditions, we started treatment with Methylprednisolone $1 \mathrm{mg} / \mathrm{kg}$ intravenous (iv) once daily. However, on the second day of hospitalization, changes on the right forearm expanded and merged with each other, making palpable, hemorrhagic, edematous plaques in different phases, on almost two thirds of the dorsal side, with pronounced swelling of dorsum of the right hand and fingers (Fig. 3). Doppler examination showed that there were regular flows through the blood vessels of the right forearm. There was no intravenous access in that arm.

In the next following days, these changes spread along the entire dorsal side of the forearm and hand, predominantly along the radial side, giving the impression of necrotic tissue, with more pronounced swelling, and with changes on the whole surface of fingers. Also, small bullous changes appeared on necrotic parts (Fig. 4). Repeated Doppler tests discovered weaker to absent vessels' flow in apical parts from the second to fourth finger, without any evidence of thrombus. All laboratory tests were repeated. CBC showed anemia (hemoglobin 58 $\mathrm{g} / \mathrm{L})$, with a decreased number of erythrocytes $\left(2.51 \times 10^{12} / \mathrm{L}\right)$ and hematocrit $(21 \%)$, which required packed red blood cell transfusion. The value of D-dimer increased up to $16.34 \mathrm{ng} / \mathrm{ml}$. All other repeated laboratory tests were within normal range.

Low-molecular-weight heparin was initiated at a dose of $100 \mathrm{IU} / \mathrm{kg}$ iv two times daily and the

Table I. Most significant laboratory parameters and comparison of patient's values along with normal values.

\begin{tabular}{|c|c|c|c|c|c|c|c|c|}
\hline $\begin{array}{l}\text { Laboratory } \\
\text { parameters }\end{array}$ & aPTT (s) & PT (s) & $\begin{array}{l}\text { Fibrinogen } \\
(\mathrm{g} / \mathrm{L})\end{array}$ & $\begin{array}{l}\text { D-dimer } \\
(\mathrm{ng} / \mathrm{ml})\end{array}$ & $\begin{array}{c}\text { Protein C } \\
(\mathrm{U} / \mathrm{mL})\end{array}$ & $\begin{array}{c}\text { Protein S } \\
(\mathrm{U} / \mathrm{mL})\end{array}$ & AT III (\%) & $(\mathrm{APCR}-\mathrm{V})$ \\
\hline Normal values & $25-35$ & $11-13$ & $2.0-4.0$ & $\geq 0.5$ & $0.72-1.23$ & $0.60-1.60$ & $80-120$ & $\geq 2.3$ \\
\hline $\begin{array}{l}\text { Patients values } \\
\text { (first / repeated) }\end{array}$ & 31.6 / 32.1 & $12.4 / 11.9$ & 4.237 / 3.926 & $\begin{array}{c}7.21 / 16.34 \text { / } \\
2.89 \text { / } 0.97\end{array}$ & $1.09 / 1.13$ & $1.26 / 1.04$ & 94.7 / 112.1 & $2.7 / 3.1$ \\
\hline
\end{tabular}




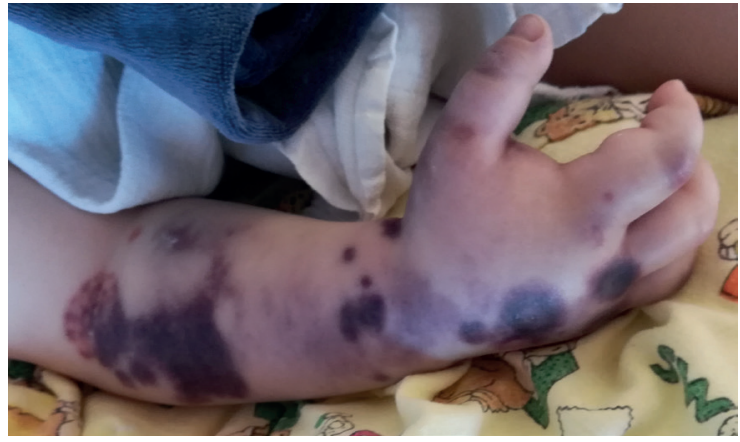

Fig. 3. Palpable, hemorrhagic, edematous changes in different phases on dorsal side of the right forearm and swelling of dorsum of the right hand and fingers.

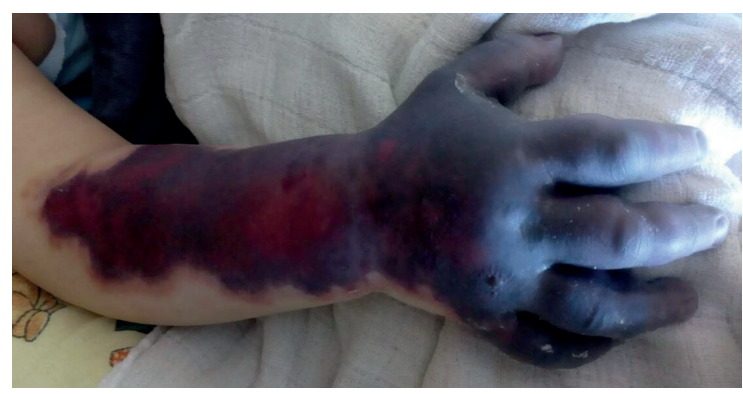

Fig. 4. Hemorrhagic changes on entire dorsal side of the right forearm, hand and whole surface of fingers, with pronounced swelling, and small bullas and necrotic parts.

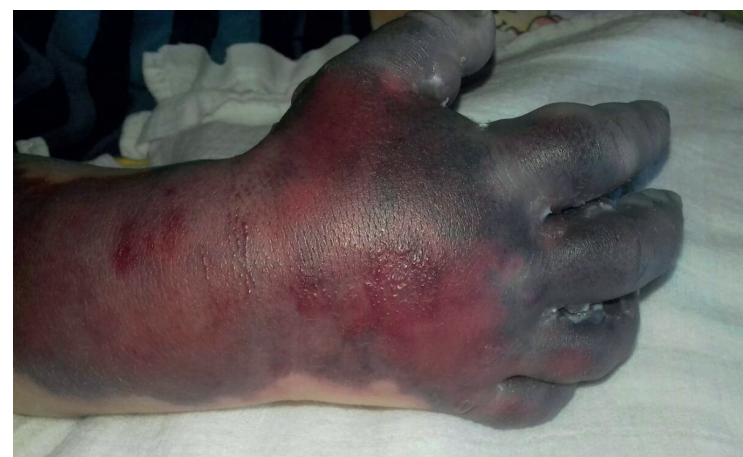

Fig. 5. Macerated bullous changes between fingers, with compartment syndrome and suspect necrosis of the proximal parts of the second to fourth finger.

dose of Methylprednisolone was increased to $1 \mathrm{mg} / \mathrm{kg}$ iv three times daily. Therapeutic option of pulsed doses of Methylprednisolone 20mg/ $\mathrm{kg}$ daily for 4 days was presented to the parents, which they refused. During hospitalization, the anti-factor Xa assays were in the therapeutic range. Also, a skin biopsy was done, and later finding confirmed nonspecific small-vessel vasculitis, with fibrin deposits in the vascular wall and perivascular infiltrates composed of neutrophils and eosinophils, with erythrocyte extravasation, again without any evidence of thrombus. On direct immunofluorescence testing, nonspecific vascular deposits of different immunoglobulins were found.

During the illness, the child was in a good general condition, afebrile, with normal laboratory analyses, after transfusion maintaining hemoglobin values around 100 $\mathrm{g} / \mathrm{L}$, with gradual decline of $\mathrm{D}$-dimer values. All other skin changes regressed, as well as changes in the oral cavity, but swelling of the right hand and fingers increased, with rising and cracking of necrotic bullous lesions. We added Ceftriaxone $70 \mathrm{mg} / \mathrm{kg}$ iv once daily to his therapy. Bullous changes between fingers occasionally macerated in close contact, movements of fingers were difficult, and that lead to the compartment syndrome with the consequent suspect necrosis of the proximal parts of the second to fourth finger (Fig. 5). On the tenth day of hospitalization, incision of bullous changes and decompression was performed, along with the necrectomy of the surface parts of the skin. All swabs taken from the wounds were sterile.

The ongoing therapy was continued, Methylprednisolone $1 \mathrm{mg} / \mathrm{kg}$ iv three times daily and low-molecular-weight heparin 100 IU $/ \mathrm{kg}$ iv two times daily, with the daily wound dressing. Distal parts of the second and third finger remained necrotic and dried eschar was formed on the dorsal side of right forearm and hand (Fig. 6). Therefore, on the 15th day of hospitalization debridement of necrotic tissue and amputation of the distal phalanges of the second and third finger was performed. After that, the child had two more operations with debridement of the remaining necrotic tissue from the forearm and hand and amputation of the middle phalanges of the second and third fingers. Afterwards, skin was transplanted from the right thigh and covered all vulnerable surfaces (Fig. 7). On regular controls, the child 


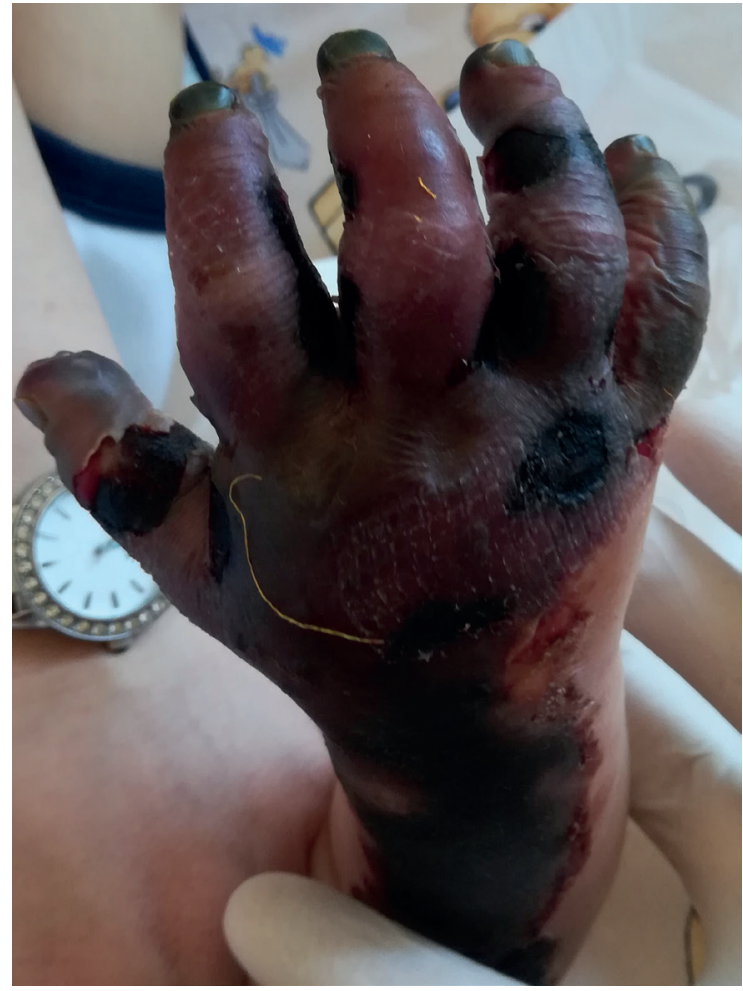

Fig. 6. Necrotic distal parts of the second and third finger and dried eschar on the dorsal side of right forearm and hand.

had no other skin changes, with adequate healing of the wound and the acceptance of the transplant after the first six months follow-up period.

Written informed consent was obtained from the parents of the children.

\section{Discussion}

AHEI was first described in 1913 by Snow ${ }^{17}$, later also named Finkelstein-Seidlmayer disease. Initially it was considered a variant of Henoch-Schönlein purpura, later however it was established that it represents a separate clinical entity. ${ }^{1,2}$ There are no reported data on the incidence, probably because of its rarity and confusion with other more common conditions. In 2008, a systematic review by Fiore et al. ${ }^{8}$ reported approximately 300 patients with AHEI, with male predominance (2:1 ratio). A review of literature by authors showed that the number of described cases increased to approximately 400

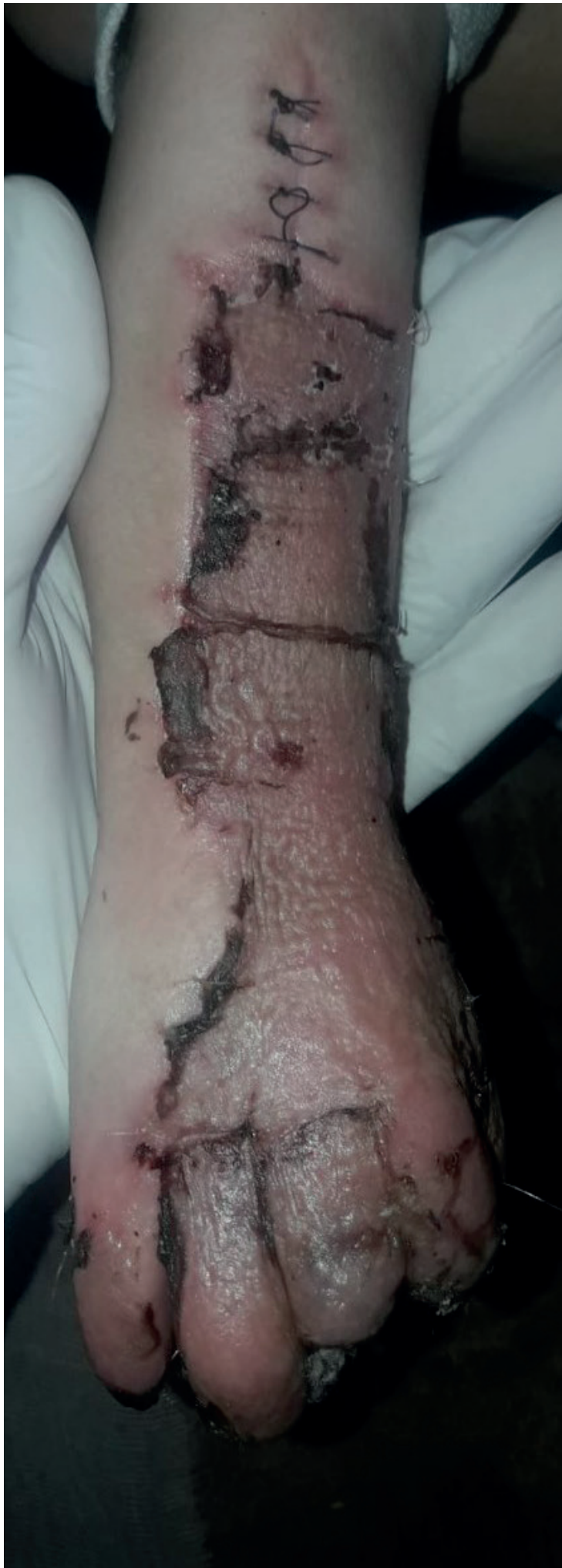

Fig. 7. Amputated distal and middle phalanges of the second and third finger and transplanted skin on the all vulnerable surfaces of the right forearm and hand. 
in the following years, probably due to better knowledge of the disease.

All authors recognized AHEI as a condition with very dramatic and impressive clinical presentation. ${ }^{2,4,7,8,10}$ Children have a prodromal illness, which is followed by the onset of palpable ecchymotic skin lesions (cockade purpura), followed by swelling. . $-5,7,8$ Lesions have a symmetrical distribution. Edema is classically asymmetrical, affecting the auricles, face and extremities (frequently dorsum of hands and feet). $\cdot^{1-4,7-10}$ Some areas may even become necrotic or bullous. ${ }^{18}$ All described cases were in good general condition. Compared with our patient, the onset of the disease clinically corresponded completely to the classic form.

AHEI has been associated with viral or bacterial infections, vaccination and drug intake. ${ }^{5-8,19}$ It occurs more during winter, which supports infectious agents as a triggering factor, according to Chesser et al. ${ }^{19}$ in $75 \%$ of cases. Another $10 \%$ of cases were connected with immunization. ${ }^{6}$ We assume that the virus that caused gingivostomatitis was responsible for initiating the immune process in our patient, but all virology examinations were negative.

The diagnosis of AHEI is initially made on clinical basis and can be made without a skin biopsy. ${ }^{1,2}$ However, a lot of diseases with similar dermatologic manifestations with potentially severe clinical presentations must be promptly differentiated (meningococcemia, skin lesions in septicemia, drug eruptions, Henoch-Schönlein purpura, Coxsackie disease, Sweet syndrome, erythema multiforme, angioedema, child abuse, idiopathic thrombocytopenia and allergies). ${ }^{1-3}$ The onset of our case was like AHEI, but in the following days, the patient's clinical picture was very similar to purpura fulminans. Unlike purpure fulminans, our patient did not develop disseminated intravascular coagulopathy, given normal fibrinogen and platelet counts over the course of the disease, also protein $C$ and $S$ values were normal, and skin biopsy did not establish the presence of thrombus in the tiny blood vessels. ${ }^{20}$
If performed skin biopsy shows leukocytoclastic vasculitis of small dermal blood vessels., ${ }^{1,25}$ According to the majority of authors, biopsy of the skin should be performed only in case of an unclear diagnosis, progression of the disease or appearance of complications. ${ }^{1,2,7,8}$ Similar opinion is also accepted for the introduction of therapy, because of the benign, self-limiting course of the disease. ${ }^{3-10}$ Risikesan et al. ${ }^{9}$ considered that rapid progression could nevertheless be an indication for steroid therapy, as we did in our case, but cited authors gave pulsed doses of Methylprednisolone $20 \mathrm{mg} / \mathrm{kg}$ daily for 4 days. With regards to our case, this was presented to the parents as a therapeutic option, which they refused, so we had to continue with $1 \mathrm{mg} / \mathrm{kg} 3$ times daily, which was obviously a mistake and did not gave a satisfactory result. According to the medical practices in Serbia, the parents, whose child is hospitalized, sign the consent to implement diagnostic procedures and they are informed about the initial therapeutic protocols. However, according to the law, they are also must be informed about any changes in the therapy during hospitalization and possible side effects. In doing so, they have the legal right to simply reject the proposed therapeutic procedure. In aforementioned case, the parents and both assigned doctors, hematologists and plastic surgeons frequently discussed the case, but parents thought that pulsed doses of Methylprednisolone would be too harmful, because they educated themselves about the benign nature of the disease. In cases with extensive bullous lesions, secondary infection of ruptured bullas must be treated locally and with systemic antibiotics. However, Lai-Cheong et al. ${ }^{18}$ advise that systemic antibiotics should nonetheless be given to combat the infection and to stop further antigenic stimulation.

In the majority of described cases, laboratory tests are generally within normal limits. We noticed that the value of D-dimer increases with the progression of AHEI, but within normal values of blood coagulation screening, fibrinogen and platelets. High activity of coagulation and fibrinolysis is associated with 
active disease of Henoch-Schönlein purpura and/or ANCA-Associated Vasculitis. ${ }^{21,22}$ The underlying mechanism is not fully clear yet. The production of the proinflammatory cytokines in active vasculitis, such as Tumor necrosis factor $(\mathrm{TNF} \alpha)$, interleukin-1 or $\mathrm{C} 5 \mathrm{a}$, may trigger thrombotic processes by increased expression of tissue factor on endothelial cell and/or neutrophils. Furthermore, markers of endothelial cell damage and apoptotic endothelial cells have been shown to become procoagulant. ${ }^{21,22}$ In the mentioned studies, the D-dimer values in the active phase of vasculitis did not increase as much as in our case. On the other hand, a study by ChaoHung $^{23}$ showed that even a very high D-dimer value was not necessarily the exclusive marker of thromboembolic disease. As there was no evidence of thrombosis in our patient, the question remains why $\mathrm{D}$-dimer values were high during disease progression and whether it could serve as some kind of prognostic parameter.

The fact is that the pathophysiological mechanism of AHEI is a release of erythrocytes through damaged blood vessels and that our patient was anemic at the onset of AHEI, however we were surprised by the drastic drop in values of hemoglobin and number of erythrocytes during deterioration of local findings on the right hand.

Very rare complications have been reported including intussusception, hematuria, proteinuria, hypocomplementemia and scarring. ${ }^{11-15}$ Other systemic symptoms such as abdominal pain, gastrointestinal bleeding, arthritis and nephritis were rarely reported as well. Severe articular involvement was described in one case, as well as testicular torsion. ${ }^{7,8,10}$ Mucosal involvement is rare, conjunctiva, palate, buccal or/and nasal mucosa were mostly affected. ${ }^{11}$ Only one case of compartment syndrome of the left foot has been described so far. Following the fasciotomy, there was marked clinical improvement in the limb perfusion. ${ }^{16}$ In our case, disease progression on the right forearm and hand was surprisingly rapid, despite the therapy initially given. Bullous changes between fingers made the compartment syndrome with the consequent necrosis of the proximal parts. Apart from the application of low-molecular-weight heparin, incision of bullas for decompression, debridement of necrotic parts and amputation of the affected fingers had to be performed. After skin transplantation and clinical improvement, both active and passive movements of the right arm and fingers were intact.

Although AHEI is a rare entity, pediatricians should be familiar with its presentation. Early recognition of AHEI is important in order to avoid unnecessary or invasive medical investigation and therapy, because of its benign nature. On the other hand, our reported case warns that unexpected complications may occur. The treatment with the corticosteroids, low-molecular-weight heparin and antibiotics in the case of extensive lesions should be considered. The $\mathrm{D}$-dimer values may be used to monitor disease progression.

\section{REFERENCES}

1. Arnold KA, Gao J, Stein SL. A review of cutaneous hypersensitivity reactions in infants: from common to concerning. Pediatr Dermatol 2019; 36: 274-282.

2. Lava SAG, Milani GP, Fossali EF, Simonetti GD, Agostoni C, Bianchetti MG. Cutaneous manifestations of small-vessel leukocytoclastic vasculitides in childhood. Clin Rev Allergy Immunol 2017; 53: 439-451.

3. Miconi F, Cassiani L, Savarese E, et al. Targetoid skin lesions in a child: acute hemorrhagic oedema of infancy and its differential diagnosis. Int J Environ Res Public Health 2019; 16: 823.

4. Halicioglu O, Akman SA, Sen S, Sutcuoglu S, Bayol U, Karci H. Acute hemorrhagic edema of infancy: a case report. Pediatr Dermatol 2010; 27: 214-2155.

5. Ferrarini A, Benetti C, Camozzi P, et al. Acute hemorrhagic edema of young children: a prospective case series. Eur J Pediatr 2016; 175: 557-561.

6. Serra E Moura Garcia C, Sokolova A, Torre ML, Amaro C. Acute hemorrhagic edema of infancy. Eur Ann Allergy Clin Immunol 2016; 48: 22-26. 
7. Parker L, Shahar-Nissan K, Ashkenazi-Hoffnung L, et al. Acute hemorrhagic edema of infancy: the experience of a large tertiary pediatric center in Israel. World J Pediatr 2017 13: 341-345.

8. Fiore E, Rizzi M, Ragazzi M, et al. Acute hemorrhagic edema of young children (cockade purpura and edema): a case series and systematic review. J Am Acad Dermatol 2008 ; 59: 684-695.

9. Risikesan J, Koppelhus U, Steiniche T, Deleuran M, Herlin T. Methylprednisolone therapy in acute hemorrhagic edema of infancy. Case Rep Dermatol Med 2014; 2014: 853038.

10. Moradinejad MH, Entezari P, Mahjoub F, Ziaee V. Acute hemorrhagic edema of infancy; a report of five Iranian infants and review of the literature. Iran J Pediatr 2011; 21: 107-112.

11. Mreish S, Al-Tatari H. Hemorrhagic lacrimation and epistaxis in acute hemorrhagic edema of infancy. Case Rep Pediatr 2016; 2016: 9762185.

12. Watanabe T, Sato Y. Renal involvement and hypocomplementemia in a patient with acute hemorrhagic edema of infancy. Pediatr Nephrol 2007; 22: 1979-1981.

13. Obeid M, Haley J, Crews J, Parhizgar R, Johnson L, Camp T. Acute hemorrhagic edema of infancy with abdominal pain and elevated transaminases. Pediatr Dermatol 2008; 25: 640-641.

14. Yu JE, Mancini AJ, Miller ML. Intussusception in an infant with acute hemorrhagic edema of infancy. Pediatr Dermatol 2007; 24: 61-64.

15. Al Sufyani MA. Acute hemorrhagic edema of infancy: unusual scarring and review of the English language literature. Int J Dermatol 2009; 48: 617-622.
16. Dotan M, Nahum E, Weigl D, Bilavsky E. Compartment syndrome because of acute hemorrhagic edema of infancy: a case report and literature review. J Pediatr Orthop B 2014; 23: 419421.

17. Snow IM. Purpura, urticaria and angioneurotic edema of the hands and feet in a nursing baby. JAMA 1913; 61: 18-19.

18. Lai-Cheong JE, Banerjee P, Hill V, Kenny P, Ross J. Bullous acute haemorrhagic oedema of skin in infancy. Clin Exp Dermatol 2007; 32: 467-468.

19. Chesser H, Chambliss JM, Zwemer E. Acute hemorrhagic edema of infancy after coronavirus infection with recurrent rash. Case Rep Pediatr 2017; 2017: 5637503

20. Canpolat C, Bakir M. A case of purpura fulminans secondary to transient protein $\mathrm{C}$ deficiency as a complication of chickenpox infection. Turk J Pediatr 2002; 44: 148-151.

21. Yilmaz D, Kavakli K, Ozkayin N. The elevated markers of hypercoagulability in children with Henoch-Schönlein purpura. Pediatr Hematol Oncol 2005; 22: 41-48.

22. Ma TT, Huang YM, Wang C, Zhao MH, Chen M. Coagulation and fibrinolysis index profile in patients with ANCA-associated vasculitis. PLoS One 2014; 9: e97843.

23. Ho $\mathrm{CH}$. Can very high level of D-dimer exclusively predict the presence of thromboembolic diseases? J Chin Med Assoc 2011; 74: 151-154. 